\title{
Understanding Clinical Complexity the Hard Way: A Primary Care Journey
}

Ross E.G. Upshur

\begin{abstract}
Ten years ago, complexity was not a term often used in primary care. In the last decade, however, the population seen in primary care has shifted, posing substantial challenges for both primary care providers and health systems. In this essay, I will document the approaches that evolved in an academic family practice environment to address the challenges posed by complex patients typified by multiple concurrent chronic conditions and social determinants challenges. I will describe the research that lead to the creation, implementation and evaluation of an interprofessional model of care and associated outcomes. I will describe how this work subsequently led to the evolution of clinical models and research projects designed to reframe the discourse around complexity as well as move forward on elaborating new policy, clinical and service delivery innovations. I will conclude with some thoughts about what I see as the major challenges in the short and immediate term for research and practice, drawing on $\mathbf{1 5}$ years of practice and research experience with complex populations.
\end{abstract}

\section{Introduction: The Emergence of Complexity}

Complexity in medical care was not discussed when I went to medical school. Indeed, the notion of complexity science was evolving in disparate research fields remote from medicine. It is only recently that the notion of complexity has emerged in analysis of both patients and the delivery of healthcare.

It is important to distinguish the concepts of complexity in healthcare from that of complexity science. Complexity science has numerous applications in a variety of fields including physics, economics, meteorology and ecology. Complexity science studies the dynamic, incompletely predictable and often chaotic behaviour of open systems.
In this essay, I focus on complexity as it relates to the understanding of patients and what makes a patient complex. Specifically, I focus on the assessment and management of complex patients in a primary care context. I draw from both clinical experience and research conducted by our team over the past decade that has focused on complex patients.

\section{What Do We Mean by Patient Complexity?}

It can be argued that the emergence of complexity in patients is primarily the result of the following driving forces:

1. Demographic transition;

2. Improved therapeutic interventions; and

3. Advent of clinical practice guidelines.

It is now well recognized that the Canadian population is aging rapidly and that the greatest gains in longevity are for those who have reached the age of 80 . It has been noted for some time that in high-income countries, the population mortality curve has become progressively rectangular. This means that the mean age of death has occurred at a progressively older age over the past half century. However, the increase in longevity has not been associated with a reduction in morbidity. In fact, the demographic transition has been associated with a growth in individuals living with multiple concurrent chronic conditions or multimorbidity. For example, Denton and Spencer (2010) show that in Canada, the number of people with no chronic conditions decreases over the life course and the co-occurrence of chronic diseases increases with age. This is often referred to as multimorbidity. It is clear now that multimorbidity is the most prevalent form of chronic disease and highly prevalent in primary care in Canada (Tinetti et al. 2010; Fortin et al. 2005). 
The idea that multimorbidity is the rule and not the exception for both population and individual health has not yet fully been integrated into health system planning and health professions education. It represents, in my mind, the pre-eminent challenge of health systems planning and health professions education in the early twenty-first century.

Improved therapy, particularly for cardiovascular conditions such as hypertension, ischemic heart disease and congestive heart failure, as well as effective therapies for cancer, have also contributed to patient complexity. Simply put, lives "saved" from fatal outcomes earlier in the life course permit the accumulation of additional morbidities through time. Ironically, better management of these diseases has prolonged life to the extent that more conditions that are chronic are acquired due to life extension. Mortality has decreased in every age category and morbidity has increased in every age category over the past half century.

Another simultaneous occurrence is the emergence and growth of clinical practice guidelines (CPGs). CPGs were virtually non-existent when I graduated from medical school in the 1980s. Under the influence of evidence-based medicine and concerns for knowledge translation, CPGs rapidly grew in the 1990s through 2000s. Weisz et al. (2007) provide a fascinating historical account that documents the impressive growth in the production of CPGs over the past 30 years. Over time, guidelines have become less opinion driven and more systematic with greater reliance on randomized controlled trials and systematic reviews. As well, the number of organizations producing CPGs has grown, and only recently has some consensus on how best to format CPGs emerged. Currently, primary care clinicians must contend with potentially thousands of CPGs. Few of these are relevant in the context of complexity (Mutasingwa et al. 2011).

The implications of the above forces became clinically relevant to me in the early 2000s. When I first started practice, as a rural family physician, in the mid-1980s, there were only a few patients in a clinic day that could be counted as having multimorbidities, and the majority of them were seniors. However, 15 years later, it was clear to me, now in an academic family practice, that my patient population had fundamentally altered in significant ways. Patients were older and typically had a higher burden of chronic disease than I had previously encountered, but such was the case for younger cohorts as well.

Part of this change was likely explained by our practice being sited in an academic tertiary care centre as well as located in an area of Toronto with a high proportion of seniors. Rather than regarding this context as a bias, it was clear that it was a microcosm of the future of primary care. If primary care were to play its proper role as the point of first contact for all patient problems and the locus of continuity of care, then it was imperative that there be the requisite management tools and care approaches to provide appropriate care for complex patients.
However, it was equally evident that primary care was not prepared for this challenge. Typical office visits in primary care are short and often do not permit attention to more than one problem at a time. This approach to care delivery in primary care sets up a situation of mutual frustration. Patients feel rushed and clinicians feel stressed. A cascade of poor quality care occurs where issues are only partially addressed.

\section{What the Literature Says About the Challenges Posed by Complex Patients}

The difficulties of managing patients with multimorbidity in primary care are numerous. The range of challenges faced by clinicians is largely because models and methods of care for this population have not evolved with the demographic transition. In essence, there is a mismatch between the needs of the patient population and how services are currently offered or funded. This mismatch is reflected in studies, indicating that primary care physicians find patients with multimorbidity "too complex and too time consuming" (Ekdahl et al. 2012).

Sinnott et al. (2013) conducted a systematic review of primary care providers' experiences of providing care to patients with multimorbidity. Four key findings emerged:

1. disorganisation and fragmentation of healthcare;

2. the inadequacy of guidelines and evidence-based medicine;

3. challenges in delivering patient-centred care;

4. barriers to shared decision-making.

They also made note of clinician's sense of isolation in decision-making for patients with multimorbidity. This isolation is a risk factor for burnout and moral distress.

Similarly Fried et al. (2011) in a study of primary care physicians' experiences of caring for older patients with multimorbidity identified the following five themes:

1. concerns about patients' ability to adhere to complex medical regimens such as lack of social supports and concerns regarding adherence;

2. variability in beliefs regarding the harms and benefits of guideline-directed care in terms of uncertainty regarding the applicability of evidence and lack of calibration of outcomes to patients' life worlds;

3. variability in approaches to balancing trade-offs between harms and benefits, such as difficulties in setting treatment priorities and the inability to quantify benefits and harms;

4. difficulties in involvement of patients in the decisionmaking process in terms of difficulty in aligning treatment goals or in conflicting goals; and

5. barriers to clinicians' preferred approaches to decisionmaking, particularly related to insufficient time and remuneration and managing unrealistic expectations. 
These findings should be taken very seriously, and policy makers and planners would do well to embark immediately on substantial reforms of service delivery and training. The situation is pressing because the demographic changes indicate a large cohort of older adults in keeping with the aging of the baby boom generation. There is every reason to believe their patterns of morbidity will match those measured in recent cohorts of older adults from the Depression and World War II generations.

\section{Quite simply, a complex patient is one whose needs exceed the temporal and informational capacity of any single provider at a particular point in time.}

\section{The Need for a Complexity Framework}

Multimorbidity is but one component of patient complexity. Schaink et al. (2012) identified additional factors that contribute to complexity. These include patterns of healthcare utilization such as high utilization, psychosocial factors such as mental health addictions and impairments of cognition, and challenges in the social determinants of health such as income, housing and food security. Many of these elements point out the current deficiencies with the way in which health and social services interact. Complex patients often have several interacting factors at play that thwart simple solutions based in medical approaches, e.g., reliance on a network of supports from family and other formal and informal caregivers.

A complexity framework should not only provide guidance in terms of explaining the nature of complexity but also be directive in helping to conceptualize the variety of care needs anticipated when seeking to provide care. Unfortunately, most clinicians and other service providers come equipped with skills that will only partially address these many needs and are often at a loss when it comes to figuring out the most appropriate manner with which to address multiple needs.

\section{Time and Information Complexity}

In my view, the essence of complexity relates to two fundamental factors: time and information. Quite simply, a complex patient is one whose needs exceed the temporal and informational capacity of any single provider at a particular point in time. Complex patients have a lot going on simultaneously. This was something I recognized in my clinical work. Patients would come with long lists of issues related to symptoms, diseases, prescriptions, the views of their specialists and things they had heard from friends and families or those they had seen on television or read on the internet pertaining to their health. The sheer volume of concerns to process in a clinical encounter far exceeded the time available for discussion. Addressing one issue at a time over a series of visits is not an optimal process, as there exists no easy way to assign priority to a particular concern and there is a high probability of one seemingly resolved condition becoming unstable in the interim.

Time is probably the most valuable of scarce resources in healthcare. Anyone with passing familiarity with modern healthcare will attest to the pervasive sense of time pressure felt by all who participate in healthcare. Patients and family members experience time stress as a sense of being rushed and express their frustration at the inability for their concerns to be adequately considered. Clinicians feel moral distress and suffer from feelings of dissatisfaction about being unable to do their jobs properly. A lack of time may be a complaint for many people, but it is particularly the case for complex patients. There is a reason behind why complex patients provoke avoidant behaviour from clinicians. Few clinicians will admit to mastery, and, as a consequence, optimal care is seldom delivered.

Richard Horton, editor of the Lancet recognized the importance of creating time-enriched environments to make complex decisions. He wrote:

However, what is the one variable - one that we have too easily given up on - that would allow these influences to be judged more carefully for the individual patient? The answer is time. Time would allow for the range of available data to be considered, and then arguments to be constructed, analysed, reviewed, refined, and finally agreed on. The approach we are currently endorsing - accepting that we must inevitably lose the fight for time, revealed by providing ever-narrower synoptic summaries or 'bottom-lines' of increasingly complex evidence - does not address the more fundamental point - namely, the need to provide a temporal space to interpret that information. (Horton 1999: 3162)

Complex patients remind us that we must be vigilant in advocating for time in order to provide appropriate highquality care. Patients accumulate chronic diseases over a life course. I would often remind medical trainees working with me that it takes a patient a long time to become complex and it will take them a long time to understand all the dimensions of their complexity. However, most healthcare professionals have not received any training with respect to the management of complex patients. This is something requiring urgent attention.

\section{Are There Any Promising Approaches?}

It was clear that current approaches were sub-optimal. With a colleague, Shawn Tracy, I argued that a new philosophy of care was required to address complexity, one that would fundamentally alter our clinical and educational approaches (Upshur and Tracy 2008). It entailed reconceptualising the 
manner in which we understand patient needs. Rather than the traditional medical approach to diagnosis and therapy based on the traditional model of seeking and reversing singular causes with the hope of cure, a model that was based in dialogue and deliberation was required.

Another dimension of complex patients is that there is often a lack of strong evidence regarding treatment and management decisions. Optimal decision-making requires acknowledgement that there is a high degree of uncertainty regarding appropriate management and prognosis associated with complex patients. If we wish to be truly patient-centred, uncertainty needs to be acknowledged. It is often the case, as a study by Kuluski and colleagues demonstrates, that patients, their caregivers and clinicians do not often share the same treatment goals (Kuluski et al. 2013). Aligning treatment goals is important to successful care planning, but often patients and families are unclear themselves about care goals or defer to healthcare professionals who are similarly confused and may devolve to acting on clinical imperatives, which may drive utilization needlessly. Investing in goal setting and goal alignment with complex patients is a time-consuming process, but a necessary step to ensure that services and needs are matched. It is also in keeping with the spirit of "patient-centred care."

\section{The Overarching Importance of Team-Based Approaches}

Recognizing these challenges, with colleagues at Sunnybrook, we created an interprofessional team-based approach to managing complex patients. Our initial model focused on older adults with multimorbidity and limitations of an activity of daily living. We designed and implemented the program in an academic family medicine environment. We have subsequently refined the model, tested it in other practices, conducted a multisite pragmatic controlled trial and adapted the model to a transition-to-home from a complex continuing-care rehabilitation setting.

We have named it the IMPACT model (Interprofessional Model of Practice for Aging and Complex Treatments) (Tracy et al. 2013). The IMPACT practice model comprises family physicians, a community nurse, a pharmacist, a physiotherapist, an occupational therapist, a dietitian and a community social worker. In addition, the model is designed to accommodate trainees from each of the various disciplines. The model was expanded in an IMPACT+ model to include specialist physicians such as those from internal medicine, psychiatry and geriatrics. One defining feature of the model is that the assessment is carried out over a period of hours, with a caregiver and all members of the team present. The virtue of concurrent assessment, compared with the sequential assessment typically conducted, is that it permits clinicians to discuss differences of opinions amongst themselves. It is often not acknowledged that the same patient being evaluated by multiple healthcare providers often receives subtly or overtly different accounts of what is wrong with them and what the optimal solution to the problem should be. The team discussions also permit healthcare professionals to express their concerns and uncertainties about the patient and the evidence that the care plan will work. The model also serves as a one-stop shop, permitting the identification of all relevant patient, provider and caregiver concerns.

Our research has shown that effective teams can be created, that healthcare providers enjoy working in this environment as it is a rich learning environment and the time afforded permits thorough reflection on the patient's problems. Patients and caregivers feel they have been given sufficient time to be heard.

I am convinced after over a decade studying this model that team-based interprofessional models are required for complex patients. There is much debate on whether this is a cost-effective model, whether it reduces utilization, particularly hospitalizations and emergency room visits. But utilization measures may not be the best measures of effect, particularly for complex patients with significant chronic disease burdens. Even with the investment of time such as in IMPACT, they will become unstable in the future.

However, the IMPACT model does help to address several of the issues identified in the literature cited above. It provides more time for all to articulate and clarify treatment goals. It helps to simplify complex treatment regimens to focus only on those issues that matter to patients and caregivers. It spares families and caregivers numerous visits for additional assessments. It provides a clinical context to acknowledge uncertainty and share the burden of decision-making. It permits the possibility for the necessary social and community services to be identified and contacted as a coordinated care plan emerges from the assessment. If for no other reason than to permit healthcare providers and caregivers to continue their struggle to provide care for people with highly demanding health and social needs, it seems more than worthwhile to me. It remains for future research to determine the optimal mix of healthcare professionals, the duration of the visit and how best to finance such models.

\section{... healthcare providers enjoy working in this environment as it is a rich learning environment and the time afforded permits thorough reflection on the patient's problems.}

\section{Concluding Thoughts}

The title of this essay refers to understanding complexity the hard way. Most of the barriers and concerns noted by primary care providers are things I have experienced in my clinical work with this population. Both clinical care and research in this area are difficult but profoundly rewarding. We are still a very long way from a health system that is optimally calibrated to manage the growing population of complex patients. We need new measures 
and management tools. A pressing need is to understand what we mean by outcomes in the context of complexity (Upshur et al. 2014). There are many signals that the broader policy and provider world is aware of these challenges, even though the focus has been disproportionately on high cost users. We need to be equally, if not more committed to quality and appropriateness of care. Innovation and research are required to address these issues. Encouraging signals such as Choosing Wisely (Leon-Carlyle et al. 2015), Minimally Disruptive Medicine (May et al. 2009), Deprescribing (Thompson and Farrell 2013) and system innovations such as Health Links in Ontario indicate the perception that there is a need to simplify and counter the belief that more assessments and more interventions will achieve meaningful clinical and patient-centred goals. My maxim: the best plan for a medically complex patient is the simplest plan to achieve and maintain the explicitly stated and agreed upon goals between patients, their caregivers and their healthcare providers. This is a tall order and much needs to be done quickly to achieve this goal including appropriate funding streams to support the work. Given the advent of the largest cohort of older adults we are likely to ever see, failure here would call into question the very meaning and purpose of a publicly funded health system. HQ

\section{References}

Denton, F.T. and B.G. Spencer. 2010. "Chronic Health Conditions: Changing Prevalence in an Aging Population and Some Implications for the Delivery of Healthcare Services." Canadian Journal on Aging 29(1): 11-21.

Ekdahl, A.W., I. Hellström, L. Andersson and M. Friedrichsen. 2012. "Too Complex and Time-Consuming to Fit in! Physicians' Experiences of Elderly Patients and Their Participation in Medical Decision Making: A Grounded Theory Study.” BMJ Open 2(3): e001063.

Fortin, M., G. Bravo, C. Hudon, A. Vanasse and L. Lapointe. 2005. "Prevalence of Multimorbidity among Adults Seen in Family Practice." Annals of Family Medicine 3(3): 223-28.

Fried, T.R., M.E. Tinetti and L. Iannone. 2011. "Primary Care Clinicians' Experiences with Treatment Decision Making for Older Persons with Multiple Conditions." American Medical Association's Journal of Internal Medicine 171(1): 75-80.

Horton, R. 2000. "Common Sense and Figures: The Rhetoric of Validity in Medicine (Bradford Hill Memorial Lecture 1999)." Statistics in Medicine 19(23): 3149-64.

Kuluski, K., A. Gill, G. Naganathan, R. Upshur, R.L. Jaakkimainen and W.P. Wodchis. 2013. "A Qualitative Descriptive Study on the Alignment of Care Goals between Older Persons with MultiMorbidities, their Family Physicians and Informal Caregivers." BMC Family Practice 14: 133.
Leon-Carlyle, M., R. Srivastava and W. Levinson. "Choosing Wisely Canada: Integrating Stewardship in Medical Education.” Academic Medicine 90(11): 1430.

May, C., V.M. Montori and F.S. Mair. 2009. "We Need Minimally Disruptive Medicine." BMJ 11: 339.

Mutasingwa, D., H. Ge and R.E.G. Upshur. 2011. "How Applicable Are Clinical Practice Guidelines to Elderly with Co-Morbidities?" Canadian Family Physician 57(7): e25362.

Schaink, A., K. Kuluski, R. Lyons, M. Fortin, A. Jadad and R. Upshur. 2012. "A Scoping Review and Thematic Classification of Patient Complexity: Offering a Unifying Framework." Journal of Comorbidity 2(1): 1-9.

Sinnott, C., S. Mc Hugh, J. Browne and C. Bradley. 2013. "GPs' Perspectives on the Management of Patients with Multimorbidity: Systematic Review and Synthesis of Qualitative Research.” BMJ Open 3(9): e003610.

Tinetti, M.E., T.R. Fried and C.M. Boyd. "Designing Health Care for the Most Common Chronic Condition--Multimorbidity." JAMA 307(23): 24934.

Tracy, C.S., S.H. Bell, L.A. Nickell, J. Charles and R.E. Upshur. "The IMPACT Clinic: Innovative Model of Interprofessional Primary Care for Elderly Patients with Complex Health Care Needs." Canadian Family Physician 59(3): e148-55.

Thompson, W. and B. Farrell. 2013. "Deprescribing: What Is It and What Does the Evidence Tell Us?" The Canadian Journal of Hospital Pharmacy 66(3): 2012.

Upshur, R.E. and S. Tracy. 2008. "Chronicity and Complexity: Is What's Good for the Diseases Always Good for the Patients?" Canadian Family Physician 54(12): 1655-58.

Upshur, R., K. Kuluski and C.S. Tracy. 2014. "Rethinking Health Outcomes in the Era of Multiple Concurrent Chronic Conditions." Healthy Debate. Retrieved July 7, 2016. <http://healthydebate. $\mathrm{ca} /$ opinions/rethinking-health-outcomes-in-the-era-of-multipleconcurrent-chronic-conditions $>$.

Weisz, G., A. Cambrosio, P. Keating, L. Knaapen, T. Schlich and V.J. Tournay. 2007. "The Emergence of Clinical Practice Guidelines." Milbank Quarterly 85(4): 691-727.

\section{About the Author \\ Ross E.G. Upshur, BA (Hons.), MA, MD, MSc, CCFP, FRCPC, is a} professor in the Department of Family and Community Medicine and the Dalla Lana School of Public Health at the University of Toronto. He is also an assistant director of the Lunenfeld Tanenbaum Research Institute in the Sinai Health System and head of the Division of Clinical Public Health at the Dalla Lana School of Public Health at the University of Toronto. He can be reached by e-mail at: ross.upshur@gmail.com. 